\title{
Quantization procedure for non-Abelian chiral bosons
}

\author{
E. Abdalla \\ Instituto de Fisica, Universidade de São Paulo, Caixa Postal 20516, São Paulo, Brazil \\ M. C. B. Abdalla \\ Instituto de Fisica Teorica, Universidade Estadual Paulista, Rua Pamplona 145 Codigo de Enderecamento Postal 01405 , \\ São Paulo, Brazil \\ (Received 18 April 1988; revised manuscript received 28 December 1988)
}

\begin{abstract}
Chiral bosonic fields are described by the Wess-Zumino-Witten action with second-class constraints. The system is quantized via the Dirac procedure, and a realization of the quantum field theory is defined by the conformal algebra. The relation to fermions is briefly discussed.
\end{abstract}

\section{INTRODUCTION}

Chiral bosonic fields are very important objects in quantum field theory. They appear naturally in string theories, ${ }^{1}$ in some integrable models, ${ }^{2}$ as well as in realistic field theories. ${ }^{3}$ Quantization of these objects may present problems and several attempts have been proposed to circumvent them. The case of a single chiral boson has been cleared up in Ref. 4. In order to put the subject in our present description, we write the action as ${ }^{5}$

$$
S=\frac{1}{2} \int d^{2} x \partial^{\mu} \phi \partial_{\mu} \phi
$$

with the chiral constraint

$$
\Omega(x)=\Pi(x)-\phi^{\prime}(x) \simeq 0,
$$

such that with the momentum definition $I I(x)=\dot{\phi}(x)$, the field $\phi(x)$ is moving to the left. The Poisson-brackets algebra is

$$
\{\Omega(x), \Omega(y)\}=-2 \delta^{\prime}(x-y) .
$$

It permits the construction of the equal-time commutator by mesons of the Dirac method: ${ }^{6}$

$$
[\phi(x), \phi(y)]_{\mathrm{ET}}=-\frac{i}{4} \epsilon(x-y) .
$$

Therefore, using the equation of motion (constraint) the whole operator content is explicitly constructed.

In this paper we generalize this construction to nonAbelian fields, considering group-valued fields $g_{i j}(x)$ described by the Wess-Zumino-Witten (WZW) action ${ }^{7,8}$ with chiral constraints. In Sec. II we develop the Dirac quantization of the system. Brief conclusions are drawn in Sec. III.

\section{DIRAC QUANTIZATION OF NON-ABELIAN CHIRAL BOSONS}

Consider a group-valued field $g_{i j}(x)$, described by the WZW action ${ }^{7,8}$

$$
\begin{aligned}
S= & \frac{1}{8 \pi} \operatorname{tr} \int d^{2} x \partial^{\mu} g \partial_{\mu} g^{-1} \\
& -\frac{1}{4 \pi} \epsilon^{\mu v} \operatorname{tr} \int d^{2} x d t g^{-1} \dot{g} g^{-1} \partial_{\mu} g g^{-1} \partial_{v} g .
\end{aligned}
$$

We may formally integrate out the auxiliary variable $t$, obtaining 9

$$
S=\operatorname{tr} \int d^{2} x\left[\frac{1}{8 \pi} \partial^{\mu} g \partial_{\mu} g^{-1}+\frac{1}{4 \pi} A(g) \partial_{0} g\right] .
$$

We do not need a complete knowledge of $A(g)$; all that is needed is its derivative

$$
F_{i j ; k l}=\frac{\partial A_{i j}}{\partial g_{l k}}-\frac{\partial A_{k l}}{\partial g_{j i}},
$$

which may be directly computed from (5), with the result ${ }^{9}$

$$
F_{i j ; k l}=\partial_{1} g_{i l}^{-1} g_{k j}^{-1}-g_{i l}^{-1} \partial_{1} g_{k j}^{-1} .
$$

We take as definition of left-moving bosons, the action (2.1) with the constraint

$$
\Omega^{L}(x)=g\left(\partial_{0} g^{-1}-\partial_{1} g^{-1}\right),
$$

while the definition of right-moving fields will be the action (2.1) with the constraint appropriately taken as

$$
\Omega^{R}(x)=\left(\partial_{0} g^{-1}+\partial_{1} g^{-1}\right) g,
$$

the difference in form of (2.5) and (2.6) being a matter of later convenience.

In order to proceed with the canonical quantization procedure we compute the momentum canonically conjugate to $g_{i j}(x)$, which is given by the expression

$$
\Pi_{i j}(x)=\frac{1}{4 \pi} \partial_{0} g_{j i}^{-1}+\frac{1}{4 \pi} A(g)_{j i} .
$$

It will be convenient to define also

$$
\widetilde{\Pi}_{i j}(x)=\Pi_{j i}(x)-\frac{1}{4 \pi} A_{i j}(g) \text {. }
$$

The fundamental Poisson brackets are postulated as usual:

$$
\begin{aligned}
& \left\{g_{i j}(x), \Pi_{k l}(y)\right\}_{\mathrm{PB}}=\delta_{i k} \delta_{j l} \delta\left(x^{1}-y^{1}\right), \\
& \left\{g_{i j}(x), g_{k l}(y)\right\}_{\mathrm{PB}}=0 .
\end{aligned}
$$

Since $\Pi_{i j}(x)$ may be realized by $-\delta / \delta g_{j i}(x)$, we have the following relations concerning the $\widetilde{\Pi}$ variable: 
$\left\{g_{i j}(x), \widetilde{\Pi}_{k l}(y)\right\}_{\mathrm{PB}}=\delta_{i k} \delta_{j l} \delta\left(x^{1}-y^{1}\right)$,

$$
\left\{\widetilde{\Pi}_{i j}(x), \Pi_{k l}(y)\right\}_{\mathrm{PB}}=-\frac{1}{4 \pi} F_{i j ; k l}(x) \delta\left(x^{1}-y^{1}\right) \text {. }
$$

We readily verify that explicit knowledge of the function $A(g)$ is not needed.

The canonical Hamiltonian is given by the expression

$$
H=\int d x^{1}\left(-2 \pi \operatorname{tr}(\widetilde{\Pi} g)^{2}+\frac{1}{8 \pi} \partial_{1} g \partial_{1} g^{-1}\right) .
$$

Again, also the Hamiltonian is independent of the topological term $A(g)$, which turns out to enter only through the momentum-momentum Poisson brackets (2.10b).

We have to rewrite the constraints in terms of the momentum, instead of the time derivative, in order to proceed with the Dirac quantization procedure. We obtain, for left and right constraints, respectively,

$$
\begin{aligned}
& \Omega_{i j}^{L}(x)=4 \pi(g \widetilde{\Pi})_{i j}(x)+\left(\partial_{1} g g^{-1}\right)_{i j}(x), \\
& \Omega_{i j}^{R}(x)=4 \pi(\widetilde{\Pi} g)_{i j}(x)-\left(g^{-1} \partial_{1} g\right)_{i j}(x) .
\end{aligned}
$$

The Poisson algebra obeyed by the constraints follows now easily from Eqs. (2.9a) and (2.9b) and reads

$$
\begin{aligned}
\left\{\Omega_{i j}^{L}(x), \Omega_{k l}^{L}(y)\right\}_{\mathrm{PB}}= & 8 \pi \delta_{i l} \delta_{k j} \delta^{\prime}\left(x^{1}-y^{1}\right) \\
+ & 4 \pi\left[\Omega_{k j}^{L}(x) \delta_{i l}-\Omega_{i l}^{L}(x) \delta_{k j}\right] \\
& \times \delta\left(x^{1}-y^{1}\right), \\
\left\{\Omega_{i j}^{R}(x), \Omega_{k l}^{R}(y)\right\}_{\mathrm{PB}}= & -8 \pi \delta_{i l} \delta_{k j} \delta^{\prime}\left(x^{1}-y^{1}\right) \\
& -4 \pi\left[\Omega_{k j}^{R}(x) \delta_{i l}-\Omega_{i l}^{R}(x) \delta_{k j}\right] \\
& \times \delta\left(x^{1}-y^{1}\right) .
\end{aligned}
$$

Taking the Poisson brackets of the Hamiltonian with $\Omega^{L}$ or $\Omega^{R}$ one readily verifies that no further constraint arises. Thus we have the $Q$ matrix $^{6}$ necessary to perform the Dirac quantization procedure

$$
Q_{i j ; k l}^{L, R}(x, y)=\left\{\Omega_{i j}^{L, R}(x), \Omega_{k l}^{L, R}(y)\right\} .
$$

The inverse in each case is given by an infinite series. They are

$$
\begin{aligned}
\left(Q^{L}\right)_{i j ; k l}^{-1}(x)= & \frac{1}{16 \pi} \delta_{i l} \delta_{k j} \epsilon\left(x^{1}\right)+\frac{1}{32 \pi}\left(\delta_{i l} \Omega_{j k}^{L}-\delta_{j k} \Omega_{l i}^{L}\right)\left|x^{1}\right|+\frac{1}{64 \pi}\left(\delta_{i a} \Omega_{j b}^{L}-\delta_{j b} \Omega_{a i}^{L}\right)\left(\delta_{a l} \Omega_{b k}^{L}-\delta_{b k} \Omega_{l a}^{L}\right) \frac{1}{2} x^{2} \epsilon\left(x^{1}\right) \\
& +\frac{1}{128 \pi}\left(\delta_{i a} \Omega_{j b}^{L}-\delta_{j b} \Omega_{a i}^{L}\right)\left(\delta_{c a} \Omega_{b d}^{L}-\delta_{b d} \Omega_{c a}^{L}\right)\left(\delta_{l c} \Omega_{d k}^{L}-\delta_{d k} \Omega_{l c}^{L}\right) \frac{1}{3} x^{3} \epsilon\left(x^{1}\right)+\cdots \\
\left(Q^{R}\right)_{i j ; k l}^{-1}(x)= & \frac{1}{16 \pi} \delta_{i l} \delta_{k j} \epsilon\left(x^{1}\right)-\frac{1}{32 \pi}\left(\delta_{i l} \Omega_{j k}^{R}-\delta_{j k} \Omega_{l i}^{R}\right)\left|x^{1}\right|-\frac{1}{64 \pi}\left(\delta_{i a} \Omega_{j b}^{R}-\delta_{j b} \Omega_{a i}^{R}\right)\left(\delta_{a l} \Omega_{b k}^{R}-\delta_{b k} \Omega_{l a}^{R}\right) \frac{1}{2} x^{2} \epsilon\left(x^{1}\right) \\
& -\frac{1}{128 \pi}\left(\delta_{i a} \Omega_{j b}^{R}-\delta_{j b} \Omega_{a i}^{R}\right)\left(\delta_{c a} \Omega_{b d}^{R}-\delta_{b d} \Omega_{c a}^{R}\right)\left(\delta_{l c} \Omega_{d k}^{R}-\delta_{d k} \Omega_{l c}^{R}\right) \frac{1}{3} x^{3} \epsilon\left(x^{1}\right)+\cdots
\end{aligned}
$$

With these expressions it is straightforward to compute the Dirac brackets. These are defined in terms of Poisson brackets by the well-known expression

$$
\{A, B\}_{\mathrm{DB}}=\{A, B\}_{\mathrm{PB}}-\left\{A, \Omega_{\alpha}\right\}_{\mathrm{PB}} Q_{\alpha \beta}^{-1}\left\{\Omega_{\beta}, B\right\}_{\mathrm{PB}} \text {. }
$$

Using (2.16) with $A$ and $B$ successively given by $g, g^{-1}$, and $\widetilde{\Pi}$, using (2.10a), (2.10b), (2.12), and (2.15), we obtain the classical Dirac algebra. In the left-moving case the result is

$$
\begin{aligned}
\left\{g_{i j}(x), g_{k l}(y)\right\}_{\mathrm{DB}}= & \pi g_{k j}(x) g_{i l}(y) \epsilon\left(x^{1}-y^{1}\right) \\
& + \text { constraints }, \\
\left\{g_{i j}(x), g_{k l}^{-1}(y)\right\}_{\mathrm{DB}}= & -\pi \delta_{i l} g_{k n}^{-1}(y) g_{n j}(x) \epsilon\left(x^{1}-y^{1}\right) \\
& + \text { constraints }, \\
\left\{g_{i j}(x), \widetilde{\Pi}_{k l}(y)\right\}_{\mathrm{DB}}= & \frac{1}{2} \delta_{i l} \delta_{k j} \delta\left(x^{1}-y^{1}\right) \\
& -\frac{1}{4} \delta_{i l}\left(\partial_{1} g^{-1}(y) g(x)\right)_{k j} \epsilon\left(x^{1}-y^{1}\right) \\
& + \text { constraints. }
\end{aligned}
$$

The result for $\{\widetilde{\Pi}(x) \widetilde{\Pi}(y)\}$ is rather long and uninteresting for our purposes. In the right-moving case we have

$$
\begin{aligned}
\left\{g_{i j}(x), g_{k l}(y)\right\}_{\mathrm{DB}}= & -\pi g_{k j}(y) g_{i l}(x) \epsilon\left(x^{1}-y^{1}\right) \\
& + \text { constraints }
\end{aligned}
$$

$$
\begin{aligned}
\left\{g_{i j}(x), g_{k l}^{-1}(y)\right\}_{\mathrm{DB}}= & \pi \delta_{k j} g_{i n}(x) g_{n l}^{-1}(y) \epsilon\left(x^{1}-y^{1}\right) \\
& + \text { constraints }, \\
\left\{g_{i j}(x), \widetilde{\Pi}_{k l}(y)\right\}_{\mathrm{DB}}= & \frac{1}{2} \delta_{i l} \delta_{k j} \delta\left(x^{1}-y^{1}\right) \\
& -\frac{1}{4} \delta_{k j}\left(g(x) \partial_{1} g^{-1}(y)\right)_{i l} \epsilon\left(x^{1}-y^{1}\right) \\
& + \text { constraints } .
\end{aligned}
$$

The "constraint" terms are infinite in number and arise from the infinite terms containing $\Omega$ in (2.15). Since our system is second class, we will eventually put $\Omega$ strongly equal to zero. Thus we do not bother about these terms.

Let us briefly discuss the Abelian case, which should appear as a particular solution. Indeed, defining, for Abelian fields,

$$
g(x)=\exp [i 2 \sqrt{\pi} \phi(x)]
$$

one easily finds that for either Poisson or for Dirac brackets, from their very definitions, the following expressions hold:

$$
\begin{aligned}
& \{g(x), g(y)\}=-4 \pi g(y) g(x)\{\phi(x), \phi(y)\}, \\
& \left\{g(x), g^{-1}(y)\right\}=4 \pi g^{-1}(y) g(x)\{\phi(x), \phi(y)\} .
\end{aligned}
$$

Thus (2.17) and (2.18) are in accordance with the Abelian case, since in our notation we have, for the Dirac brackets, 


$$
\begin{aligned}
& \left\{\phi^{L}(x), \phi^{L}(y)\right\}=-\frac{1}{4} \epsilon\left(x^{1}-y^{1}\right), \\
& \left\{\phi^{R}(x), \phi^{R}(y)\right\}=\frac{1}{4} \epsilon\left(x^{1}-y^{1}\right) .
\end{aligned}
$$

In the Abelian case the infinite constraint terms are absent in (2.17) and (2.18).

Because of the nonlocality of the Dirac brackets in (2.17)-(2.19), substitution of them by commutators in order to define a quantum theory, is an ambiguous procedure, due to the ordenation problem in the right-hand side. However, we can take the Abelian case as a valuable hint towards a solution to our problem; in that situation, quantization has been achieved promoting (2.23) to $i / \hbar$ times commutators, to obtain (upper sign corresponds to left constraints and the lower sign to right ones)

$$
[\phi(x), \phi(y)]_{\mathrm{ET}}= \pm \frac{i \hbar}{4} \epsilon\left(x^{1}-y^{1}\right) .
$$

Thus, building the quantum analogous of (2.20), i.e., $g(x)=: \exp [i 2 \sqrt{\pi} \phi(x)]:$, one finds

$$
[g(x), g(y)]=g(x) g(y)\{\exp [ \pm i \hbar \pi \epsilon(x)]-1\},
$$

which gives (2.17) as a classical limit. Thus one learns that it is worth working with elements of the algebra, rather than working with elements of the group.

In the right-moving case we start out of

$$
\begin{aligned}
J_{-i j}(x) & =-\frac{i}{4 \pi}\left(g \partial_{-} g^{-1}\right)_{i j}(x) \\
& =\left[-i g \widetilde{\Pi}+\frac{i}{4 \pi} g \partial_{1} g^{-1}\right](x),
\end{aligned}
$$

which, using the constraint $(2.12 b)$, is weakly equal to

$$
J_{-i j}(x) \simeq \frac{i}{2 \pi}\left(g \partial_{1} g^{-1}\right)_{i j}(x) .
$$

It corresponds, in the Abelian case, to the minus derivative of $\phi(x)$ : namely, $\partial_{-} \phi(x)$.

Using the Dirac algebra (2.17b), (2.18b), and (2.19b) we find, for right movers,

$$
\begin{aligned}
\left\{J_{-}^{a}(x), J_{-}^{b}(y)\right\}= & f^{a b c} J_{-}^{c}(x) \delta(x-y) \\
& -\frac{1}{2 \pi} \delta^{a b} \delta^{\prime}(x-y),
\end{aligned}
$$

which can be readily quantized, since only linear terms show up in the right-hand side, and there are no longer ambiguities

$$
\left[J_{-}^{a}(x), J_{-}^{b}(y)\right]=i f^{a b c} J_{-}^{c}(x) \delta(x-y)-\frac{i}{2 \pi} \delta^{a b} \delta^{\prime}(x-y) .
$$

Since we have a second-class system, it is perfectly legitimate to take the constraint strongly $\Omega_{i j}^{R}(x)$ equal to zero, implying that the left current

$$
J_{+i j}(x)=\left[-i \widetilde{\Pi} g+\frac{i}{4 \pi} g^{-1} \partial_{1} g\right]_{i j}(x)
$$

is strongly equal to zero. The Hamiltonian is of the Sugawara form; furthermore, in order to have the usual form of the Kac-Moody algebra with unity central charge, ${ }^{10}$ we fix the coefficient of the Hamiltonian

$$
H_{-}=\frac{\pi}{c_{V}+1} \int d x^{1}:\left[J_{-}^{a}(x)\right]^{2}: .
$$

A generalization of the quantum theory to the case $k \neq 1$ (central charge of the Kac-Moody algebra not unity) is straightforward, the only difference being the coefficient of $H_{-}$, which turns out to be $\pi /\left(c_{V}+k\right)$.

The equation of motion for $J_{-}^{a}(x)$ is subtle, due to normal-order problem in (2.31). Following Refs. 10 and 11 , one sees that the coefficient of the quantum Hamiltonian in (2.31) exactly cancels in the commutator $\left[H_{-}, J_{-}\right]$, and one obtains

$$
i \dot{J}_{-}^{a}(x)=\left[J_{-}^{a}(x), H_{-}\right]=-i J_{-}^{a \prime}(x),
$$

which is the desired equation of motion. Together with the constraint, taken strongly as $J_{+}=0$ we have a theory of right-moving non-Abelian chiral fields.

We may analogously start out of $J_{+}^{a}(x)$, obtaining the Dirac algebra for left movers

$\left\{J_{+}^{a}(x), J_{+}^{b}(y)\right\}=f^{a b c} J_{+}^{c}(x) \delta(x-y)+\frac{1}{2 \pi} \delta^{a b} \delta^{\prime}(x-y)$

with analogous formulas for (2.29), (2.31), and (2.32) (which reads $\partial_{-} J_{+}=0$ ) with the constraint $J_{-}(x) \simeq 0$.

\section{CONCLUSIONS}

From the definition of $J$, Eq. (2.26), we have a differential equation which must be obeyed by the field $g$. That system has been extensively discussed in Ref. 11, and correlators involving $g(x)$ may be computed using their methods. Each sector, namely, + or -, is a representation of a Kac-Moody-Virasoro algebra. We have consistently constrained the field of the WZW theory either to the right- or to the left-moving sector. Each model is such that the Hamiltonian is of the Sugawara form, with a current obeying a Kac-Moody algebra with unity extension.

These results generalize those of Floreanini and Jackiw $^{4}$ to the non-Abelian case. Nonetheless, it is not possible in our case to compute arbitrary commutators, as done in Ref. 4. The result is analogous to the interaction of chiral fermions with gauge fields: in the Abelian case, it is possible to compute arbitrary commutators (see, e.g., Ref. 12), while in the non-Abelian case, the same procedure as a whole turns out to be unfeasible (see Ref. 9 as an example).

It is worthwhile to mention again that the direct quantization of the fundamental fields presents ambiguities due to a nonlocal candidate to an "equal time commutator" of the fundamental Lagrangian fields.

On the other hand, we achieved quantization of the algebra-valued field, while group-valued objects were computed through differential equations, where the algebra-valued fields entered as a quantum external potential in the differential equations. Those may be solved in terms of known functions. As an output we obtained a 
bosonic chiral representation of fermions from the nonAbelian Thirring model, since those objects obeyed the same set of differential equations. This can be interpreted as a generalization of the non-Abelian bosonization procedure to chiral fields, such that the bosonic field has a correlator which may be directly related to the known fermionic correlator. ${ }^{13-15}$

\section{ACKNOWLEDGMENTS}

This work was partially supported by Conselho $\mathrm{Na}$ cional de Desenvolvimento Científico e Tecnológico, Brazil.
${ }^{1}$ D. J. Gross, J. A. Harvey, E. Martinec, and R. Rohm, Nucl. Phys. B256, 253 (1985); B267, 75 (1986).

2J. L. Gervais and A. Neveu, Nucl. Phys. B199, 59 (1982); E. Abdalla, and A. Lima-Santos, Mod. Phys. Lett. A 3, 1579 (1988).

${ }^{3}$ S. Treiman, R. Jackiw, B. Zumino, and E. Witten, Current Algebra and Anomalies (Princeton University Press, Princeton, NJ/World Scientific, Singapore, 1985).

${ }^{4}$ R. Floreanini and R. Jackiw, Phys. Rev. Lett. 59, 1873 (1987).

5J. M. F. Labastida and M. Pernici, Nucl. Phys. B297, 557 (1988); Phys. Rev. Lett. 59, 2511 (1987).

${ }^{6}$ P. A. M. Dirac, Lectures in Quantum Mechanics (Yeshiva University, New York, 1964).

${ }^{7}$ A. Polyakov and P. B. Wiegman, Phys. Lett. 131B, 121 (1983).

${ }^{8}$ E. Witten, Commun. Math. Phys. 92, 455 (1984).

${ }^{9}$ E. Abdalla and K. D. Rothe, Phys. Rev. D 36, 3190 (1987).
${ }^{10} \mathrm{P}$. Goddard and D. Olive, Int. J. Mod. Phys. A 1, 303 (1986).

${ }^{11}$ V. G. Knizhnik and A. B. Zamolodchikov, Nucl. Phys. B247, 83 (1984).

${ }^{12}$ H. O. Girotti, H. J. Rothe, and K. D. Rothe, Phys. Rev. D 33, 514 (1986).

${ }^{13}$ Belavin, A. M. Polyakov, and A. B. Zamolodchikov, Nucl. Phys. B 241, 333 (1984).

${ }^{14}$ E. Abdalla and M. C. B. Abdalla, Rev. Bras. Fis (to be published); E. Abdalla, Bosonization Methods in String Theory, Meeting on High Energy Physics, Valparaiso, Chile, 1987 [Scientia (Valparaiso) 165-166, 3 (1987)]; Thirring Strings: Use of Generalized Non-Abelian Bosonization Techniques, Meeting on Fundamental Problems, Santiago, Chile, 1987 (Plenum, New York, in press).

${ }^{15}$ R. Dashen and Y. Frishman, Phys. Rev. D 11, 2781 (1975). 